\title{
Condensation Transition in DNA-Polyaminoamide Dendrimer Fibers Studied Using Optical Tweezers
}

\author{
F. Ritort, ${ }^{1,4}$ S. Mihardja, ${ }^{3}$ S. B. Smith,,${ }^{1,2}$ and C. Bustamante ${ }^{1,2}$ \\ ${ }^{1}$ Department of Physics and Molecular \& Cell Biology, University of California, Berkeley, California 94720, USA \\ ${ }^{2}$ Howard Hughes Medical Institute, University of California, Berkeley, California 94720, USA \\ ${ }^{3}$ Department of Chemistry, University of California, Berkeley, California 94720, USA \\ ${ }^{4}$ Department of Physics, Faculty of Physics, University of Barcelona, Diagonal 647, 08028 Barcelona, Spain
}

(Received 11 October 2004; published 22 March 2006)

\begin{abstract}
When mixed together, DNA and polyaminoamide dendrimers form fibers that condense into a compact structure. We use optical tweezers to pull condensed fibers and investigate the decondensation transition by measuring force-extension curves (FECs). A characteristic force plateau (around $10 \mathrm{pN}$ ) and hysteresis between the pulling and relaxation cycles are observed for different dendrimer sizes, indicating the existence of a first-order transition between two phases (condensed and extended) of the fiber. Upon salt variation FECs change noticeably confirming that electrostatic forces drive the condensation transition. We propose a simple model for the decondensing transition that qualitatively reproduces the FECs and which is confirmed by atomic force microscopy images.
\end{abstract}

DOI: $10.1103 /$ PhysRevLett.96.118301

PACS numbers: 82.35. $-\mathrm{x}, 82.37 .-\mathrm{j}, 87.15 .-\mathrm{v}$

Introduction.-In eukaryotes, genomic DNA is compacted in a complex nucleoprotein phase known as chromatin. Such condensation is an important mechanism for protecting the genetic information from external factors, as well as in storing the long DNA into a compartment with dimensions on the order of microns. The basic unit of chromatin organization is a nucleosome. There 8 histone molecules join to form a core particle $\sim 6 \mathrm{~nm}$ in diameter and the DNA wraps $\sim 2$ times around that core forming a solenoid that is $10 \mathrm{~nm}$ wide. When such particles are spaced evenly along the DNA, they form the $10 \mathrm{~nm}$ fiber or beads on a string structure as seen in electron micrographs. Under various salt conditions, nucleosomes prefer higher-order condensed structures such as a $30 \mathrm{~nm}$ fiber, $100 \mathrm{~nm}$ fiber, etc. It has long been recognized that the main driving force for condensation is electrostatic interaction between negative DNA and positive histone proteins [1] but little is known about what determines the stability of these specific structures. Do the size and shape of core particle determine how DNA wraps around it and thus the amount of length compaction? Are specific charge patches on the core particles required for DNA winding or would a uniform spherical charge distribution work as well? What factors determine spacing between nucleosomes in the $10 \mathrm{~nm}$ fiber? In order to answer such questions it may be useful to consider model systems that are much simpler than the DNA-histone complexes while, at the same time, retaining some of the important characteristics of the DNA-histone fibers.

To this end, we have chosen to study the condensation of DNA by polyaminoamide (PAMAM) dendrimers. These dendrimers are synthetic branched polymers that exhibit such excellent properties as molecular uniformity, welldefined size, high water solubility, and very low toxicity. Because of these special properties, PAMAM dendrimers are currently being investigated for their potential in such biological applications as the transfection of foreign genetic materials into eukaryotic cells [2]. The PAMAM dendrimers we investigated were synthesized via an initiator core EDA, ethylenediamine, followed by a series of repeated polymerization steps (the number of steps defines the generation $G$ of the dendrimer), terminating with amino $\left(\mathrm{NH}_{2}\right)$ groups. At physiological $p \mathrm{H}$ values, the surface amino groups of the dendrimers are protonated. To study the structure of the condensed state and to elucidate the effects of dendrimer charge and size, we pulled DNA-dendrimer fibers using optical tweezers. Based on our results, we propose a simple theoretical model for the condensation-decondensation transition that qualitatively reproduces the force-extension curves (FECs). This model is further validated via atomic force microscopy (AFM) imaging of the DNA-dendrimer complexes. Finally, we compare our results with those of optical-tweezers experiments done on native chicken erythrocyte chromatin fibers [3] and on reconstituted chromatin fibers [4,5].

Experimental setup. - Pulling experiments were carried out with a fragment of $\lambda$-phage DNA [6] and three dendrimer generations $G 5, G 6, G 8$. Here, the number of surface amino groups equals 128,256 , and 1024 and the diameters equal 5.7, 6.7, and $9.7 \mathrm{~nm}$, respectively [7]. Most of the experiments were done in buffer containing $100 \mathrm{mM}$ of $\mathrm{NaCl}$. We used optical tweezers to exert force on the DNA molecule as shown in Fig. 1(a). Two types of polystyrene beads (Spherotech) of $3 \mu \mathrm{m}$ in diameter were used: streptavidin (SA) coated beads and antidigoxigenin (AD) beads [8]. SA beads were held in a dual-beam optical trap [9]. A tether was made by fishing for the free end of the DNA with an AD bead kept on a micropipette by suction. After first characterizing the properties of the naked DNA, the chamber $(30 \mu \mathrm{l})$ was rinsed with $100 \mu \mathrm{l}$ of buffer 


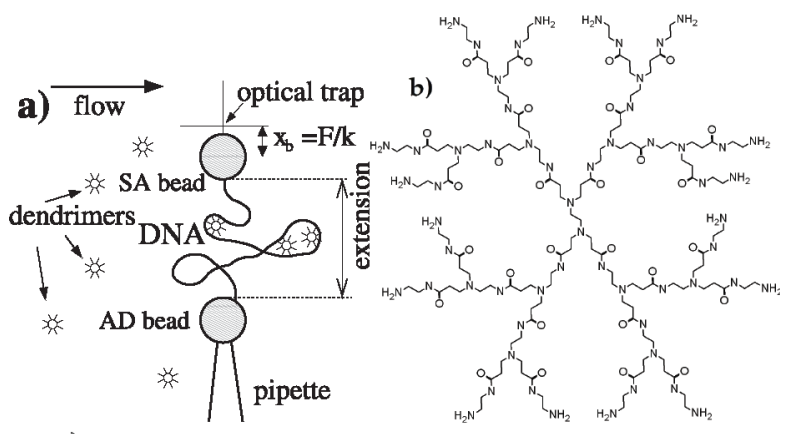

c)

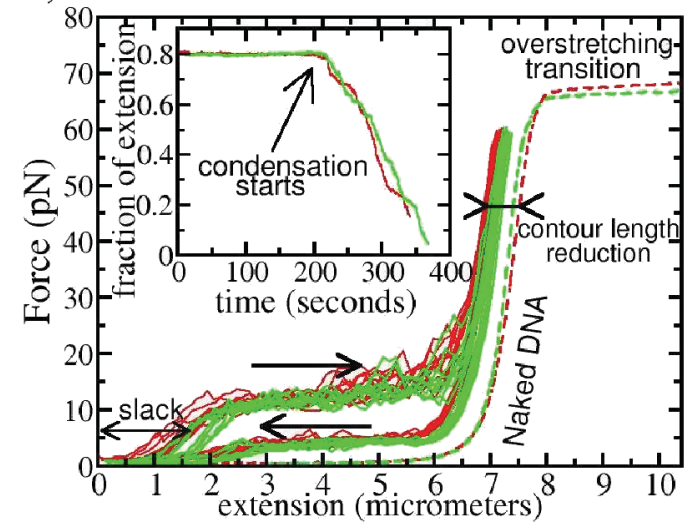

FIG. 1 (color). (a) Experimental setup and (b) the chemical structure of a dendrimer molecule $G 2$ with 16 amino terminal groups. (c) Reproducibility of FECs using CFP for two DNA molecules (red and green colors) that were condensed with $G 8$ dendrimers under the same conditions at a constant force of $4 \mathrm{pN}$. Dashed curves correspond to naked DNA. Inset: Relaxation curves during the condensation of the fiber. The arrow indicates the time at which condensation starts.

containing $\sim 30 \mathrm{nM}$ of dendrimers. This process typically lasted $8 \mathrm{~min}$. The flow was then halted and the fiber was repeatedly pulled by moving a piezo-controlled stage attached to the pipette.

DNA was condensed by one of two protocols: (1) constant-extension protocol (CEP) and (2) constantforce protocol (CFP). In the CEP, the distance between the center of the optical trap and the bead on the tip of the micropipette was kept constant while the chamber was rinsed with the buffer containing dendrimers. As the dendrimers bound to the DNA, the force increased with time. In the CFP, a feedback loop mechanism was used to maintain a constant force. Here, condensation was marked by a decrease in extension of the molecule as a function of time.

Results. - FECs are reproducible on different molecules if they are condensed following the same protocol and exposed to the dendrimers under identical conditions. This effect is shown in Fig. 1(c) where two different DNA molecules, condensed at constant force under identical conditions, generated the same FEC. The FECs show the existence of a well-defined force plateau along the pulling curve, centered about $10 \mathrm{pN}$. This feature is the signature of a condensation-decondensation first-order phase transition separating a condensed $(C)$ from an extended $(E)$ phase. Upon relaxation, the fiber once again undergoes a transition from the $E$ to the $C$ form that manifests as a lower force plateau. The FECs display the following general features (for both protocols and for all dendrimer generations): (a) strong hysteresis between the pulling and relaxation curves; (b) repeatable pattern of FECs over successive pulling-relaxation cycles; (c) shortening of the contour length of the extended fiber $(\simeq 5 \%)$ suggesting that dendrimers remain bound to the DNA in the $E$ phase; (d) existence of a "slack" region in the FECs at short end-to-end extensions, where the fibers extend by $\sim 1 \mu \mathrm{m}$ at relatively low $(<3 \mathrm{pN})$ force.

When the protocol conditions differ, the FECs change from experiment to experiment. In general, for all condensation protocols, the amount of slack is anticorrelated to the reduction observed in the effective contour length of the fiber (i.e., the observed extension where the force rises rapidly in the $E$ phase). A larger slack is always accompanied by a smaller reduction in contour length, suggesting that the origin of the slack is the presence of naked DNA regions. Using different time protocols, we verified that the amount of slack depends on the time exposure of the fiber to the dendrimer flow. A large slack is observed when a fiber is exposed to flow for a short time $(\sim 5 \mathrm{~min})$ whereas for a longer exposure $(\sim 30 \mathrm{~min})$ the slack became too short to measure accurately $(<0.1 \mu \mathrm{m}$, data not shown). Oddly, the measured slack decreased only while the dendrimer buffer was flowing past the fiber but not as the buffer sat quiescent in the chamber. After the flow had stopped, the FECs remained repeatable in time. This effect probably indicates a rapid partition of available dendrimers between the DNA and glass chamber walls, and a depletion of dendrimers in the buffer. Similar depletion effects have also been observed for positively charged proteins that stick to negative glass walls.

To check whether dendrimers exchanged between the DNA and those in the buffer solution during the pullingrelaxation cycle, we flowed clean dendrimer-free buffer solution through the center of the chamber and repeatedly pulled the fiber several times in the presence of a small flow to wash out any dendrimers expelled from the backbone. No time-dependent change in the FEC was observed. Evidently, dendrimers are irreversibly bound to the DNA backbone, i.e., they do not bind and unbind from the DNA during the pulling cycles. This fact was further tested by trying to condense DNA while keeping the fiber at forces as high as $20 \mathrm{pN}$. Condensation was observed in all cases indicating that dendrimers bind to DNA up to very high forces. In contrast, real chromatin fibers eventually lose their histone proteins after repeated pulls or when subjected to high forces and eventually assume the FECs characteristic of naked DNA [4,5].

For identical condensation protocols, FECs are affected by dendrimer size in a systematic manner (Fig. 2 upper 
panel). The larger the generation is, the higher the value of the decondensing force plateau. This result may be attributed to the increase in electrostatic strength, i.e., increase in surface amino groups, with increasing dendrimer generation.

The importance of the electrostatic interaction as the main force stabilizing the condensed state is revealed by investigating the effect of salt concentrations on the FECs (lower panel in Fig. 2). Clearly, as the salt concentration increases, both the extent of hysteresis and the value of the decondensing force plateau decrease. At high salt concentrations $(500 \mathrm{mM} \mathrm{NaCl})$ the hysteresis disappears and the FEC shows a plateau at low forces $(\sim 1 \mathrm{pN})$ similar to that observed when DNA condenses in the presence of multivalent cations [10]. At low salt concentrations the hysteresis increases noticeably and the pulling curve becomes noisy, revealing a stickier fiber (uppermost curve in Fig. 2, lower panel).

The model. - Because DNA and dendrimers have opposite charge, dendrimers will bind nonspecifically and will distribute themselves nonhomogeneously along the length of the DNA backbone. In certain regions in which many dendrimers bind side by side, the DNA tends to condense forming a compact globular structure ( $C$ phase) which is maintained by electrostatic forces bridging dendrimers with nonadjacent segments of DNA [11]. These globular

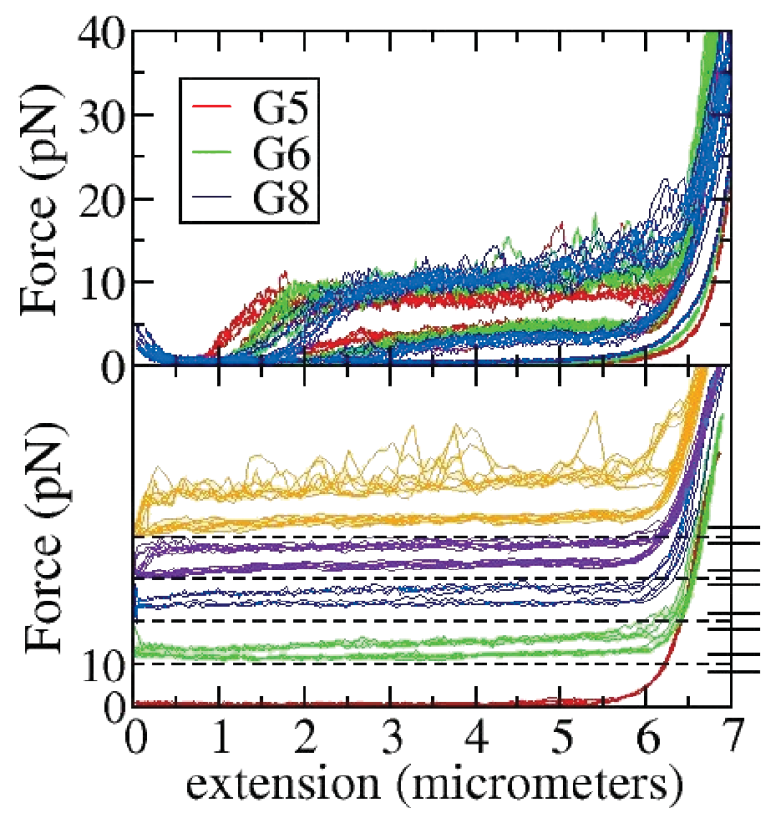

FIG. 2 (color). Upper panel: Size dependence of FECs using CFP for different dendrimer sizes. The dashed curves are naked DNA. All extensions have been normalized to a common contour length. Lower panel: Salt dependence of FECs in $G 5$ fibers. From top to bottom 10 (orange), 50 (indigo), 100 (blue), 200 (green), 500 (red) $\mathrm{mM} \mathrm{NaCl}$. For the sake of clarity FECs corresponding to different salt concentrations have been shifted $10 \mathrm{pN}$ upwards in the figure. structures are interspersed with regions of naked DNA. When the fiber is pulled, initially work is done to straighten the regions of naked DNA (entropic elasticity) giving rise to the observed slack. As these naked regions are straightened and the force reaches a certain critical value (the value of the plateau) the bridges that hold together the globular structures begin to yield resulting in their mechanical decondensation, revealed by the plateau in the FECs. At the end of this process, all globular structures have been converted to an extended phase ( $E$ phase) in which the DNA molecule resembles a line of negative charge with a random sequence of positively charged patches along its backbone. Such extended structure might conceivably resemble the $10 \mathrm{~nm}$ fiber in chromatin. This model and the existence of the $C$ and $E$ phases were confirmed by AFM images of the fibers (Fig. 3, insets) [12].

Finally, we have considered a simple theoretical description of our model that can qualitatively reproduce the experimental results shown in Fig. 1, in particular, the force plateau, the large hysteresis observed during the pulling-relaxation cycle, and the qualitative difference between the pulling curve (showing a plateau) and the relaxation curve (showing a soft shoulder). The model consists of a collection of $N$ noninteracting two-level systems that mimic the contacts between dendrimers and neighboring DNA segments. The parameters describing these molecular contacts are the free-energy of formation of each $i$ th contact $\Delta G_{i}$, its activation barrier $B_{i}$ and the distance $\Delta x_{i}^{\dagger}$ to the contact-formed state. Upon the action of mechanical force each contact yields an extension $x_{i}$ in a

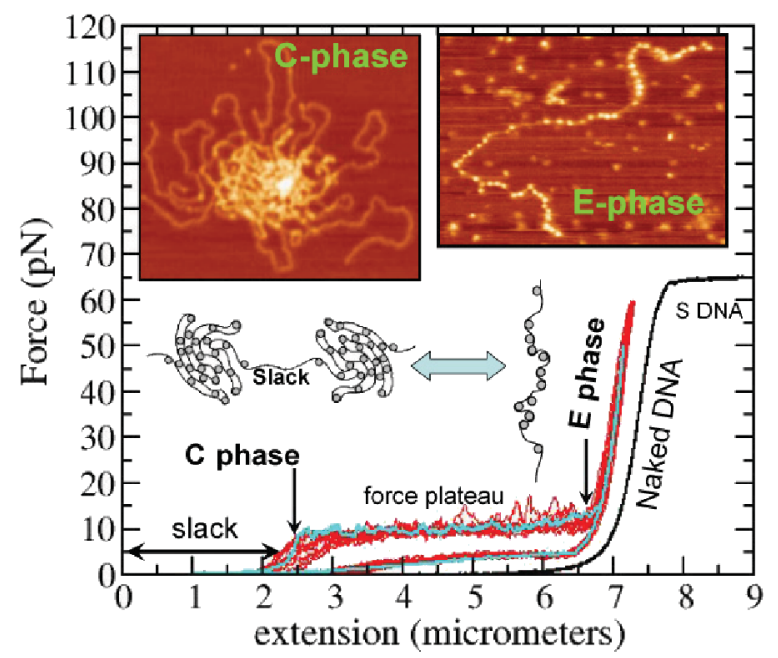

FIG. 3 (color). Main features of FECs and schematic model for the distinct phases $(C, E)$ of the DNA-dendrimer fibers. The red curve corresponds to DNA condensed with $G 6$ in the CFP at a force of $8.4 \mathrm{pN}$. The black curve corresponds to naked DNA. The cyan curve is the prediction of the theoretical model (see the text). Vertical arrows denote $100 \%$ of the corresponding phases. The schematic model has been checked by taking AFM images (G5) showing the formation of different structures ( $C, E$ phases). 
thermally activated process [13]. The resulting model shows qualitative agreement with the experimental results by including some structural disorder (Fig. 3, blue curve) [14]. In general, a few hundred contacts are needed to reproduce the experimental data implying that a similar number of dendrimers are adsorbed onto the DNA backbone.

Conclusion. - We have explored the behavior of DNA condensed by PAMAM dendrimers in hopes of finding a simple model to explain chromatin condensation. Our results do show some similarities between the DNAdendrimer complex and chromatin. Under certain conditions, FECs show similar unfolding and refolding forces and have similar compaction ratios to chromatin fibers [35]. However, it may be possible that nonspecific condensation of DNA by other polycationic complexes (e.g., eukaryotic condensin, spermidine [15]) also exhibit similar behavior.

An essential aspect of chromatin structure is the fact that DNA wraps $\sim$ twice around each histone complex. We have little evidence that something similar happens for dendrimers. Our AFM images cannot resolve the path of the DNA around or through such particles. Slack in the condensed fiber disappears with sufficient exposure to dendrimers. Thus it probably does not represent a stable extended state like the $10 \mathrm{~nm}$ chromatin fiber. Previous optical-tweezers pulling experiments on reconstituted chromatin $[4,5]$ revealed a "sawtooth" force pattern corresponding to sudden opening of the DNA wraps. Except for a few cases (with $G 8$ ), we never clearly identified such events. Perhaps backfolding of the terminal amino groups inside the dendrimer and the considerable flexibility of low generation dendrimers [16] allow the dendrimers to bend around the DNA more than the DNA bends around the dendrimers. Such flexibility would help explain the tight binding of dendrimers to DNA at high tension $(\leq 60 \mathrm{pN})$, where hard spheres would lose contact with a linear molecule pulled straight [17]. The structural deformation of dendrimers bound to DNA might be confirmed by NMR, TEM, or $a b$ initio numerical simulations of DNAdendrimer complexes. Further experiments using varied salt, temperature, and $p \mathrm{H}$ conditions should allow for considerable refinement of the dendrimer-DNA model. It might be worthwhile to carry out pulling experiments with more rigid condensing agents such as metallic gold nanoparticles to simulate nucleosomes.

We acknowledge stimulating discussions with Dr. Jean M. J. Frechet and assistance with DNA preparation by Dr. Pan Li. This work was supported in part by the U.S. Department of Energy Grant No. DE-AC0376SF00098, GTL2BB "Microscopy of Molecular Machines," the Spanish research council (FIS2004-3454) and the Catalan government (Distinció de la Generalitat).
[1] T.D. Yager, C. T. McMurray, and K.E. van Holde, Biochemistry 28, 2271 (1989).

[2] J.F. Kukowska-Latallo et al., Proc. Natl. Acad. Sci. U.S.A. 93, 4897 (1996); A. Bielinska et al., Nucleic Acids Res. 24, 2176 (1996).

[3] Y. Cui and C. Bustamante, Proc. Natl. Acad. Sci. U.S.A. 97, 127 (2000).

[4] M. L. Bennink et al., Nat. Struct. Biol. 8, 606 (2001).

[5] B. D. Brower-Toland et al., Proc. Natl. Acad. Sci. U.S.A. 99, 1960 (2002).

[6] For the optical-tweezers experiments, a 24-kbp $\lambda$-DNA fragment (New England Biolabs) was made by digesting the linear DNA at the Xba I site. The DNA was labeled on one end with biotin and on the other end with digoxigenin.

[7] PAMAM dendrimers were purchased from Dendritic Nanotechnologies, Inc. For the single-molecule experiments, the dendrimers were first diluted $1 / 100$ with water and then another $1 / 1000$ to $10 / 1000$ by TE buffer $[10 \mathrm{mM}$ Tris- $\mathrm{HCl} p \mathrm{H}$ 7.5, $1 \mathrm{mM}$ EDTA, $100 \mathrm{mM} \mathrm{NaCl}, 1.5 \mathrm{mM}$ $\mathrm{MgCl} 2,0.02 \%(v / v)$ Tween 20 , and $0.01 \%(w / v)$ milk powder].

[8] SA beads were incubated with DNA at concentrations such that on average there was $<1$ DNA molecule per bead. AD beads were made by cross-linking polyclonal sheep anti-digoxigenin (Roche) to Protein G beads (Spherotech).

[9] S. B. Smith, Y. Cui, and C. Bustamante, Methods Enzymol. 361, 134 (2003).

[10] C. G. Baumann et al., Proc. Natl. Acad. Sci. U.S.A. 94, 6185 (1997).

[11] R. Netz and J.-F. Joanny, Macromolecules 32, 9026 (1999); H. Boroudjerdi and R. Netz, Europhys. Lett. 64, 413 (2003).

[12] For the AFM experiments, a $3.5 \mathrm{kbp}$ DNA fragment was incubated with dendrimers at a 4:1 (DNA phosphate:Dendrimer amine) ratio for $5 \mathrm{~min}$ at room temperature in $10 \mathrm{mM}$ Tris- $\mathrm{HCl}(p \mathrm{H} \mathrm{7.5}), 1 \mathrm{mM}$ EDTA, $100 \mathrm{mM} \mathrm{NaCl}, 1.5 \mathrm{mM} \mathrm{MgCl}_{2}$. AFM images were obtained using the Nanoscope IIIA Scanning Probe Microscope (Digital Instruments).

[13] G. I. Bell, Science 200, 618 (1978); E. Evans, Annu. Rev. Biophys. Biomol. Struct. 30, 105 (2001).

[14] Experimental Setup: $k=0.1 \mathrm{pN} / \mathrm{nm}, k_{B} T=4.11 \mathrm{pN} \mathrm{nm}$, pulling speed $200 \mathrm{~nm} / \mathrm{s}$, bead radii $1 \mu \mathrm{m}$; DNA fiber: slack $2.7 \mu \mathrm{m}$, E-DNA contour and persistence lengths are 7.2 and $20 \mathrm{~nm}$, respectively, Young modulus $Y_{\mathrm{E}-\mathrm{DNA}}=$ $2000 \mathrm{pN}$; Two-state contacts: total number $=300, \Delta x_{i}^{\dagger}=$ $3 \mathrm{~nm}, x_{i}=15 \mathrm{~nm}$, critical force at equilibrium $f_{c}=7 \mathrm{pN}$ and dissociation rate at $f_{c}, k_{c}=0.01 \mathrm{~Hz}$. Free-energies are exponentially distributed $P(\Delta G)=(1 / w) \exp \left(\left(\Delta G_{0}-\right.\right.$ $\Delta G) / w$ ) with $\Delta G \geq \Delta G_{0}=20 k_{B} T$ and $w=10 k_{B} T$.

[15] C. G. Baumann et al., Biophys. J. 78, 1965 (2000).

[16] P. K. Maiti, T. Cagin, G. Wang, and W. A. Goddard III, Macromolecules 37, 6236 (2004).

[17] J.F. Marko and E.D. Siggia, Biophys. J. 73, 2173 (1997). 